\title{
Editorial "Sarcopenia"
}

\section{Cornel C. Sieber}

Department of Internal Medicine - Geriatrics and Institute for Biomedicine of Aging, Friedrich Alexander University Erlangen-Nuremberg, Nuremberg, Germany

The demographic shift poses challenges to the Health system on a broad scale. Frailty and its pathophysiological twin - sarcopenia - are especially important as the loss of fat free mass ( $\mathrm{sar} x=$ flesh) goes along with a decrease in functionality, thereby loss of independence and finally drop of quality of life.

In this issue, three different articles track the actual knowledge in this fast emerging field both with regard to diagnostics and therapeutical possibilities. Michael Drey starts the triad by commenting upon the pathophysiology and clinical relevance of frailty. Dorothée Volkert then comments on nutritional therapeutic interventions, whereas Ellen Freiberger outlines the different concepts as well as therapeutic options with regard to physical activity programs. It has to be stressed that effective treatment strategies in sarcopenia always include both nutritional and physical activity interventions in parallel.

It is intended to provide the interested reader with the essential background, but also with practical recommendations to help the fast growing elderly population at stake.

\section{Conflict of interest}

The author declares that there is no conflict of interest. 